\title{
Utilização da planta Stryphnodendron adstringens (mart.) Coville na cicatrização de
}

\section{feridas: um estudo etnobotânico}

\author{
Use of the plant Stryphnodendron adstringens (mart.) Coville in wound cicration: an etnobotanical
} study

Utilización de la planta Stryphnodendron adstringens (mart.) Coville para la cicración de heridas: un estudio etnobotánico

Dailon de Araújo Alves ORCID: https://orcid.org/0000-0001-8294-298X Faculdade de Medicina Estácio de Juazeiro do Norte, Brasil E-mail: dailon.araujo12@gmail.com

Rosa Maria Grangeiro Martins ORCID: https://orcid.org/0000-0002-2516-0719 Faculdade de Medicina Estácio de Juazeiro do Norte, Brasil

E-mail: rosamaria13gm@gmail.com

Cícera Rejane Tavares de Oliveira ORCID: https://orcid.org/0000-0002-3379-4738 Prefeitura Municipal de Juazeiro do Norte, Brasil

E-mail: rejane.ta@hotmail.com

Janayle Kéllen Duarte de Sales ORCID: https://orcid.org/0000-0003-0894-2070 Universidade Regional do Cariri, Brasil E-mail: rejane.ta@hotmail.com

Francisca Clarisse de Sousa ORCID: https://orcid.org/0000-0002-9429-3777 Universidade Regional do Cariri, Brasil E-mail: clarisse.sousa@urca.br

Jackeline Kérollen Duarte de Sales ORCID: https://orcid.org/0000-0002-5953-5697 Universidade Regional do Cariri, Brasil E-mail: jackelinekerollen@hotmail.com

Gledson Micael da Silva Leite

ORCID: https://orcid.org/0000-0002-7656-7084 Universidade Regional do Cariri, Brasil E-mail: gledson.micael@urca.br

Ariadna Moisés do Nascimento Arrais Ribeiro ORCID: https://orcid.org/0000-0001-5576-3441

Hospital e Maternidade São Camilo, Brasil E-mail: ariadna_arrais@hotmail.com

Leiliane de Queiroz Oliveira

ORCID: https://orcid.org/0000-0001-8020-4641 Faculdade de Medicina Estácio de Juazeiro do Norte, Brasil E-mail: leilianenfermeira@ hotmail.com

Regina Petrola Bastos Rocha ORCID: https://orcid.org/0000-0003-0626-232X Faculdade de Medicina Estácio de Juazeiro do Norte, Brasil E-mail:rpetrola7@gmail.com

Antônio Ygor Modesto de Oliveira ORCID: https://orcid.org/0000-0002-3650-5997 Universidade Regional do Cariri, Brasil E-mail: ygormodesto.saude@gmail.com

Raquel Duarte Pereira

ORCID: https://orcid.org/0000-0002-5485-5077

Hospital São Raimundo, Brasil E-mail: duartepereiraraquel@gmail.com

Maria de Lourdes Silva

ORCID: https://orcid.org/0000-0002-9151-5071 


\author{
Maria Niná Morais Tavares \\ ORCID: https://orcid.org/0000-0001-7347-3712 \\ Hospital e Maternidade São Camilo, Brasil \\ E-mail: mninatmoraais2016@ hotmail.com \\ Luis Rafael Leite Sampaio \\ ORCID: https://orcid.org/0000-0003-1437-9421 \\ Universidade Regional do Cariri, Brasil \\ E-mail:rafael.sampaio@urca.br
}

\begin{abstract}
Resumo
Objetivo: Conhecer a utilização etnobotânica e tradicional do barbatimão no estabelecimento do processo cicatricial de feridas. Metodologia: Trata-se de uma revisão integrativa da literatura, descritiva e com abordagem qualitativa. A mesma foi realizada em agosto de 2019, com os seguintes descritores DeCS e MeSH, wound healing, woundsand injuries, Stryphnodendron adstringens; intercalados pelo operador booleano AND. A busca ocorreu via Portal de Periódicos da Capes, nas seguintes bases de dados: MEDLINE, LILACS, BDENF, CINAHL, COCHRANE LIBRAY, IBECS, SCIENCE DIRECT. Após realização da busca nas bases de dados, obteve-se 15 artigos, que segundo a aplicação de critérios de inclusão e exclusão, restaram apenas, três estudos que atendiam ao objeto de pesquisa em estudo. Resultados: Os resultados apontaram que os estudos tem em comum a realização de levantamentos etnobotânicos, destacando o uso tradicional por meio do conhecimento popular, de diversas espécies vegetais, e dentre elas, o barbatimão apresentou um grande destaque entre os estudos selecionados, principalmente no que diz respeito ao uso como elemento cicatrizador de feridas cutâneas. Considerações finais: A utilização dessa planta ainda é pouco difundida no meio científico no processo de cicatrização de ferimentos e lesões, mesmo com o conhecimento popular acerca de sua aplicabilidade, os estudos pouco tratam a preparação, concentração, tempo de uso, interações com outras medicações e efeitos adversos acerca do barbatimão, dificultando sua avaliação quanto sua eficácia terapêutica.
\end{abstract}

Palavras-chave: Cicatrização de feridas; Feridas e lesões; Barbatimão.

\begin{abstract}
Objective: To know the ethnobotanical and traditional use of barbatimão in the establishment of the wound healing process. Method: This is an integrative literature review, descriptive and with a qualitative approach. It was conducted in August 2019, with the following DeCS and MeSH descriptors, wound healing, woundsand injuries, Stryphnodendron astringens; interspersed by the Boolean operator AND. The search was conducted via the Capes Periodicals Portal, in the following databases: MEDLINE, LILACS, BDENF, CINAHL, COCHRANE LIBRAY, IBECS, SCIENCE DIRECT. After conducting the search in the databases, 15 articles were obtained, which according to the application of inclusion and exclusion criteria, remained only, three studies that met the research object under study. Results: The results pointed out that the studies have in common the realization of ethnobotanical surveys, highlighting the traditional use through popular knowledge, of several plant species, and among them, the barbatimão presented a great prominence among the selected studies, mainly regarding the use as a healing element for skin wounds. Final considerations: The use of this plant is still little diffused in the scientific environment in the process of healing wounds and injuries, even with the popular knowledge about its applicability, the studies deal little with the preparation, concentration, time of use, interactions with other medications, and adverse effects on barbatimão, making it difficult to evaluate its therapeutic efficacy.
\end{abstract}

Keywords: Wound healing; Woundsand injuries; Stryphnodendron adstringens.

\title{
Resumen
}

Objetivo: Conocer la utilización etnobotánica y tradicional del barbatimón en el establecimiento del proceso cicatricial de las ferias. Método: Se trata de una revisión bibliográfica integradora, descriptiva y con un enfoque cualitativo. Se realizó en agosto de 2019, con los siguientes descriptores DeCS y MeSH, wound healing, woundsand injuries, Stryphnodendron astringens; intercalados por el operador booleano AND. La búsqueda se realizó a través del Capes Periodicals Portal, en las siguientes bases de datos: MEDLINE, LILACS, BDENF, CINAHL, COCHRANE LIBRAY, IBECS, SCIENCE DIRECT. Después de realizar la búsqueda en las bases de datos, se obtuvieron 15 artículos, que de acuerdo a la aplicación de los criterios de inclusión y exclusión, quedaron solamente, tres estudios que cumplían con el objeto de investigación en estudio. Resultados: Los resultados señalaron que los estudios tienen en común la realización de encuestas etnobotánicas, destacando el uso tradicional a través del conocimiento popular, de diversas especies vegetales, y entre ellas, el barbatimão presentó un gran protagonismo entre los estudios seleccionados, principalmente en lo que respecta al uso como elemento curativo de heridas cutáneas. Consideraciones finales: El uso de esta planta es todavía poco difundido en el medio científico en el proceso de curación de heridas y lesiones, incluso con el conocimiento popular sobre su aplicabilidad, los estudios tienen poco que ver con la preparación, la concentración, el tiempo de uso, las interacciones con otros medicamentos, y los efectos adversos en barbatimão, lo que hace difícil evaluar su eficacia terapéutica.

Palabras clave: Curación de heridas; Heridas y lesiones; Barbatimão. 


\section{Introdução}

As lesões podem ser definidas como alterações das estruturas da pele e são classificadas segundo o agente causador, em: processos cirúrgicos, traumas, infecções, substâncias tóxicas, isquemia, pressão, doenças autoimunes, insuficiência arterial ou venosa. Dessa forma, há uma grande prevalência de lesões em pacientes acompanhados em instituições hospitalares, o que representa enormes gastos, aumento do tempo de internação e morbimortalidade dos pacientes acometidos, acarretando um substancial problema de saúde pública (Passaretti, et al., 2016).

Por sua vez, alguns tipos de lesões podem implicar na formação de feridas, as quais são representadas pela interrupção da continuidade de um tecido corpóreo, em maior ou menor extensão, oriunda de qualquer trauma de ordem física, química, mecânica ou desencadeada por uma afecção clínica. Elas podem ser agrupadas em dois tipos básicos: agudas ou crônicas. As feridas denominadas como agudas, estão associadas ao processo de cicatrização não complicado, organizado e habitualmente rápido à restauração da integridade funcional e anatômica. Já as feridas crônicas são tidas como qualquer alteração nas funções anatômicas e fisiológicas dos tecidos, complicadas pela presença de uma ou mais doenças crônicas, com tempo de duração superior a três meses, tendo assim, um processo de cicatrização prolongado (Ramalho, et al., 2018).

Dessa forma, existem diversos tipos de tratamentos direcionados para o cuidado de feridas, sendo um deles, a utilização de plantas medicinais ou extratos de origem vegetal. Segundo Wang et al., (2013), mediante a grande quantidade de recursos existentes, as plantas medicinais, em geral de baixo custo e facilmente encontradas nos mais variados ambientes, ganham espaço no mercado com a finalidade aumentar as opções terapêuticas.

Há grande variedade de espécies de plantas na Terra com potencial terapêutico/medicinal, e somente uma pequena parte desse montante é utilizada para o tratamento de doenças. De acordo com Veiga Júnior, (2008), não são muitas as pesquisas científicas no Brasil, que avaliam o grau de utilização das plantas como medicamentos e sua cultura popular em relação a outros países, com incipiente conhecimento dos profissionais de saúde sobre a cultura de utilização das plantas medicinais pela população.

De certa forma, as plantas medicinais são indicadas e utilizadas após a formação de um ferimento cutâneo, por serem a elas atribuídas propriedades terapêuticas. Algumas evitam a formação de processos infecciosos, promovem a coagulação do sangue e até alcançam a cicatrização funcional e satisfatória; representando assim, em excelentes benefícios para o quadro clínico e restabelecimento sistêmico do paciente (Souza \& Rodrigues, 2016).

Assim, existe no meio vegetal uma planta muito utilizada para o tratamento de feridas, conhecida popularmente por barbatimão, barba-de-timão, casca da virgindade, barbatimão-branco, faveira, casca da mocidade, enche-cangalha. Além disso, trata-se de uma espécie amplamente distribuída pelas cinco regiões brasileiras (Cardoso, et al., 2016). Da família das Fabaceae, subfamília Mimosoideae, apresenta nomenclatura botânica de Stryphnodendron adstringens(Mart.) Coville, todavia, pode ser identificada também, por sinonímias botânicas, com as seguintes designações: Acaciaadstringens Mart., Mimosa barbadetimam Vell., Mimosa virginalis Arruda, Stryphnodendron barbatimão Mart. e S. barbatimam(Vell.) Mart. (Brasil, 2014).

Ela é considerada uma planta perenifólia, com altura variando de 2 a 8 metros, o tronco apresenta uma tortuosidade de 20 a $30 \mathrm{~cm}$ de diâmetro, sendo a parte interna do cerne vermelha, rígida e possui troncos com cascas rugosas e rígidas que se desprendem facilmente. As folhas são bipinadas e a floração ocorre no período de setembro a novembro, período esse, em que brotam pequenas flores de coloração creme-esverdeadas, assentadas em inflorescências que possuem formato de espiga. Já o processo de frutificação, ocorre entre os meses de novembro a junho, os frutos são vagens cilíndricas de 6 a 9 cm de comprimento, com várias sementes de coloração verde (imaturos) ou parda (maduros) (Lima, et al., 2016).

Vale destacar que as cascas dessa planta são ricas em tanino, flobafenos e glicídio solúvel e apresentam efeitos adstringentes. Os taninos condensados estimulam o processo cicatricial, uma vez que se ligam as proteínas dos tecidos lesados, 
criando uma camada protetora que isola o local da ferida, reduzindo a permeabilidade e exsudação da ferida, promovendo a reparação dos tecidos. Os taninos também possuem atividades vasoconstritoras e anti-inflamatórias, estimulam o crescimento da epiderme, auxiliando na reepitelização, além de apresentar ações antimicrobianas e antiulcerogênicas (Passaretti, et al., 2016).

Conforme corroboram Eurides, et al., (1996) a ação farmacológica dessa planta como cicatrizante de feridas e úlceras se deve a riqueza dos taninos, bem como, as propriedades adstringentes do barbatimão possibilitam que as proteínas das células superficiais da mucosa e dos tecidos lesados desprotegidos se precipitem, formando um revestimento protetor contra a multiplicação bacteriana, promovendo uma ação anti-séptica.

Segundo Ramalho, et al., (2018), outra propriedade cicatrizante que o barbatimão possui está relacionada com o fato de estimular a multiplicação de queratinócitos, que estão ao redor da área lesionada, facilitando assim, a reepitelização da ferida.

O barbatimão é muito utilizado como antisséptico, anti-inflamatório, hemostático, antiedematogênico, antioxidante, antidiabético, anti-hipertensivo, adstringente, analgésico, cicatrizante, antimicrobiano e no tratamento de diversas infecções de origem cutânea (Pereira, Moreno \& Carvalho, 2013).

Essa espécie foi incluída na lista da Relação Nacional de Plantas Medicinais de Interesse ao SUS (RENISUS), mediante a realização de diversos estudos etnobotânicos e a confirmação de sua eficácia terapêutica, além de ser recomendada no formulário de fitoterápicos da Farmacopeia Brasileira, como um elemento cicatrizante na forma farmacêutica de creme (Farmacopeia Brasileira, 2011).

Dessa forma, a realização desse estudo surge como uma necessidade de se desvendar e aprimorar cada vez mais, o conhecimento acerca dos produtos naturais, com ênfase nos alicerces etnobotânicos, e avaliação do seu potencial benefício para a terapêutica de pacientes detentores de lesões na pele. Além do que, pesquisas nesse segmento, ainda pouco exploradas, possibilitam ao meio científico, a apropriação de um conhecimento mais aprofundado, crítico e diagnóstico; possibilitando assim, mudanças na maneira de assistir aos pacientes.

Por sua vez, o objetivo primordial desse estudo é conhecer, por meio de uma revisão integrativa da literatura, a utilização etnobotânica e tradicional do barbatimão no estabelecimento do processo cicatricial de feridas.

\section{Metodologia}

Trata-se de uma revisão integrativa da literatura, descritiva e com abordagem qualitativa, de artigos acerca do uso da planta barbatimão na cicatrização de ferimentos e lesões.

Esse método de pesquisa literária é utilizado na Prática Baseada em Evidências (PBE), que permite a incorporação das evidências no contexto da prática clínica, além de sintetizar e reunir resultados de pesquisas acerca de um determinado tema ou questão, de forma sistemática e ordenada, contribuindo para o aprofundamento do conhecimento do tema investigado (Mendes, Silveira \& Galvão, 2008).

Para consolidação do presente método se faz necessário o seguimento de seis etapas, a saber: formulação da questão norteadora e/ou problemática do estudo, pesquisa e seleção dos estudos, recolhimento e processamento dos dados da investigação, análise crítica dos resultados, interpretação e/ou síntese dos resultados, e apresentação (Souza, Silva \& Carvalho, 2010).

Inicialmente elaborou-se a seguinte questão de pesquisa: Como ocorre a utilização da planta barbatimão na cicatrização de feridas e lesões? Essa questão foi construída de acordo com a estratégia Population, Variables and Outcomes (PVO), observando seu contexto e suas variáveis, sendo P: Planta barbatimão, V: Feridas e lesões O: Cicatrização. 
Na segunda etapa, ocorreu a busca dos artigos via Portal de Periódico da Capes, com a utilização dos seguintes descritores, sendo os dois primeiros citados em termos MeSH (Medical Subject Heading) e o último, em DeCS (Descritores em Ciências da saúde): Wound healing, Woundsand injuries, Stryphnodendron adstringens. Os termos foram intercalados pelo operador booleano AND.

As bases, portais e bibliotecas, compreenderam as seguintes: Cochrane Library (The Cochrane Central Register of Controlled Trials - CENTRAL), Science Direct, LILACS (Literatura Latino-Americana e do Caribe em Ciências da Saúde), MEDLINE (Medical Literature Analysis and Retrieval Sistem Online), IBECS (Indice Bibliográfico Espanõl em Ciencias de laSalud), CINAHL (Cummulative Index to Nursing and Allied Health Literature), BDENF (Base de Dados de Enfermagem), SciELO(Scientific Electronic Library Online). Ressalta-se que a busca do material bibliográfico ocorreu em agosto de 2019, de maneira pareada. A Tabela 1, expressa o cruzamento dos descritores nas respectivas bases de dados.

Tabela 1. Estratégia de busca dos artigos por meio do cruzamento dos DeCS e MeSH nas bases de dados.

\begin{tabular}{cc}
\hline Bases de dados & Estratégia \\
\cline { 2 - 3 } & Wound healing AND Woundsand injuries \\
& Stryphnodendron adstringens \\
\hline Science Direct & 10 \\
\hline LILACS & 3 \\
\hline MEDLINE & 1 \\
\hline CINAHL & 1 \\
\hline BDENF & 0 \\
\hline Cochrane Library & 0 \\
\hline IBECS & 0 \\
\hline Total & $\mathbf{1 5}$ \\
\hline
\end{tabular}

Fonte: Autores.

Para a seleção dos artigos utilizou-se os seguintes critérios de inclusão: (a) Artigos originais; (b) artigos disponibilizados na íntegra; (c) estudos relacionados ao uso do barbatimão na cicatrização de ferimentos e lesões cutâneas; (d) estudos observacionais (analíticos e descritivos); (e) estudos publicados em inglês, português ou espanhol.

Foram aplicados os seguintes critérios de exclusão: (a) Estudos duplicados ou que não abordem temática relevante ao alcance do objetivo da revisão; (b) Teses, dissertações, monografias, relatos de experiência, anais de eventos científicos, por meio da leitura do título e resumo na íntegra, conforme expresso na Figura 1. Ressalta-se que não houve delimitação temporal. 
Research, Society and Development, v. 10, n. 15, e394101522688, 2021

(CC BY 4.0) | ISSN 2525-3409 | DOI: http://dx.doi.org/10.33448/rsd-v10i15.22688

Figura 1. Fluxograma da seleção dos estudos de acordo com o Preferred Reporting Items for Systematic Reviews and Meta-

Analyses (PRISMA).

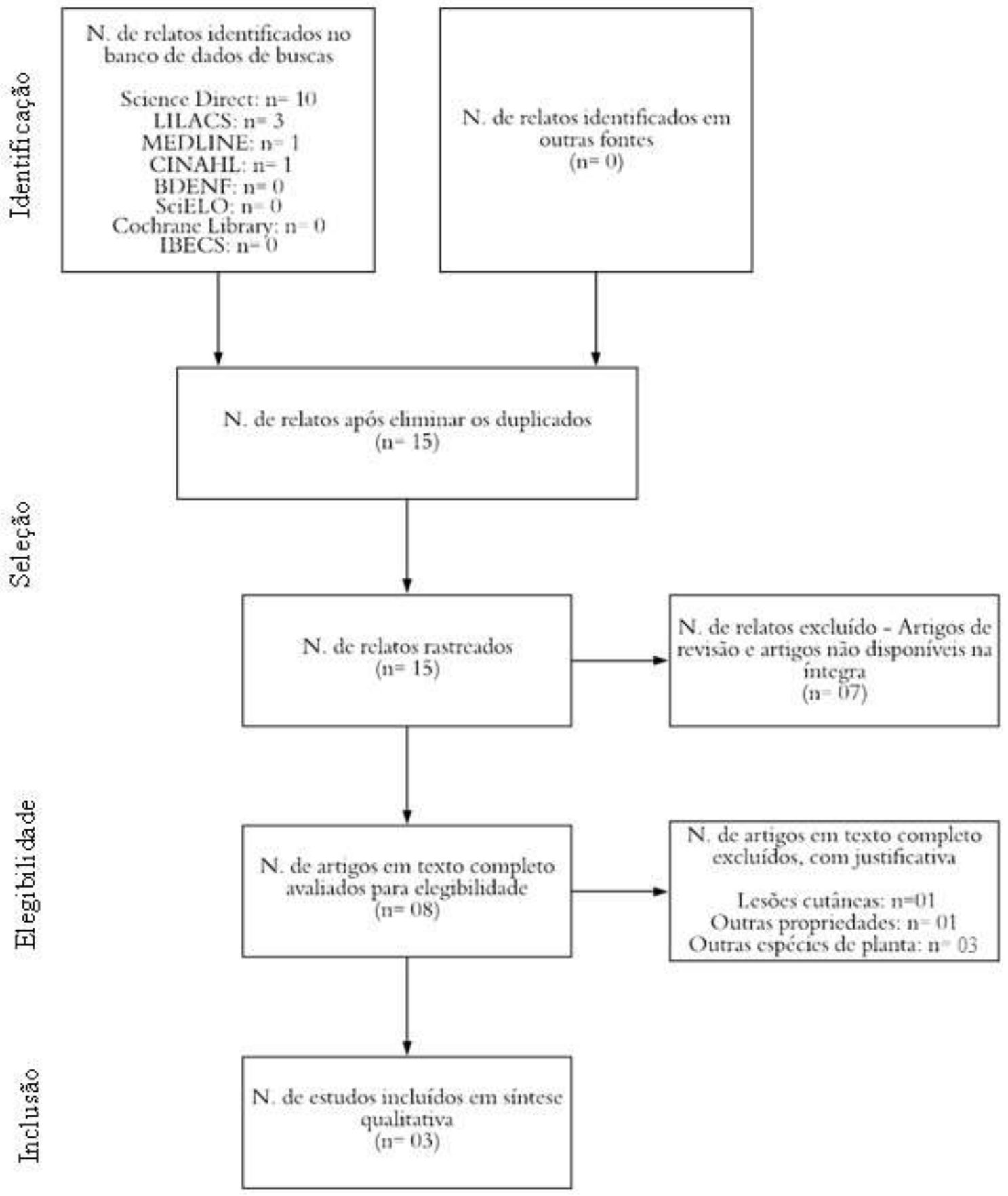

Fonte: Autores.

\section{Resultados}

Após aplicação da estratégia de busca juntamente com os critérios de inclusão, conforme demonstrado na Figura 01, a amostra final deste estudo resultou em três (03) artigos, os quais sintetizaram os principais resultados sobre o uso do barbatimão no processo de cicatrização de feridas, conforme exposto no Quadro 1. 
Quadro 1. Síntese dos artigos incluídos na revisão integrativa.

\begin{tabular}{|c|c|c|c|}
\hline AUTOR E ANO & $\begin{array}{c}\text { TIPO } \\
\text { ESTUDO }\end{array}$ & OBJETIVO & PRINCIPAIS ACHADOS \\
\hline $\begin{array}{l}\text { Albuquerque, et } \\
\text { al., (2006) }\end{array}$ & $\begin{array}{c}\text { Estudo } \\
\text { Etnobotânico }\end{array}$ & $\begin{array}{l}\text { Comparar a riqueza taxonômica das plantas } \\
\text { vendidas no local em diferentes anos. } \\
\text { Investigar diferenças entre a importância } \\
\text { relativa das espécies de plantas e apresentar } \\
\text { descrições de seus principais usos. }\end{array}$ & $\begin{array}{l}\text { O barbatimão é utilizado para tratamento de } \\
\text { inflamações, déficit da circulação, cicatrização de } \\
\text { feridas, limpeza de feridas uterinas. }\end{array}$ \\
\hline $\begin{array}{l}\text { Bieski, et al., } \\
\text { (2015) }\end{array}$ & $\begin{array}{c}\text { Estudo } \\
\text { Etnobotânico }\end{array}$ & $\begin{array}{l}\text { Levantar, identificar, catalogar e } \\
\text { documentar as espécies de plantas } \\
\text { medicinais utilizadas no vale do Juruena, no } \\
\text { noroeste do Mato Grosso. }\end{array}$ & $\begin{array}{l}\text { O barbatimão é usado como agente } \\
\text { antimicrobiano, na cicatrização de feridas e no } \\
\text { tratamento do diabetes, leishmaniose, úlceras } \\
\text { pépticas, infecção uterina, problemas vaginais, } \\
\text { inflamação, fratura óssea, infecção da bexiga e } \\
\text { infecção nos rins. }\end{array}$ \\
\hline $\begin{array}{l}\text { Ribeiro, et al., } \\
\text { (2017) }\end{array}$ & $\begin{array}{c}\text { Estudo } \\
\text { Etnobotânico }\end{array}$ & $\begin{array}{l}\text { Identificar e documentar espécies de plantas } \\
\text { medicinais utilizadas por especialistas } \\
\text { locais no norte do Araguaia, Mato Grosso. }\end{array}$ & $\begin{array}{l}\text { O barbatimão é usado para o tratamento de } \\
\text { candidíase, infecções, doença venérea, erisipela, } \\
\text { micose ungueal, seborreia, hemorragia, } \\
\text { hemorroidas, dor estomacal, gastrite, úlcera, } \\
\text { calvície, inflamação, reumatismo, corrimento } \\
\text { vaginal, infecção uterina, infecção urinária, } \\
\text { infecção vaginal com corrimento, inflamação } \\
\text { uterina e ovariana, inflamação vaginal, cálculos } \\
\text { renais, ferida infectada, infecção na garganta, } \\
\text { inflamação na garganta e na cicatrização de } \\
\text { feridas. }\end{array}$ \\
\hline
\end{tabular}

Fonte: Autores.

Frente aos resultados obtidos com o estudo, por meio dos artigos selecionados e analisados, averiguou-se de forma geral, que os estudos evidenciam que o barbatimão apresenta efeitos benéficos no processo de cicatrização, e o conhecimento popular acerca de suas propriedades cicatrizantes é difundido em diferentes locais do país.

A partir da análise minuciosa dos artigos foi averiguado que o barbatimão é utilizado no tratamento de vários processos fisiopatológicos decorrentes de enfermidades bacterianas, virais e fúngicas, dentre os quais podemos citar principalmente o uso do barbatimão na cicatrização de feridas.

\section{Discussão}

A cicatrização de feridas é um evento complexo, sistêmico e dinâmico. Este processo se configura como uma cascata coordenada de eventos a níveis bioquímicos, moleculares e celulares (Medeiros \& Dantas-Filho, 2017).

Dentre as alterações ocorridas no tecido lesionado, a fibroplasia (aumento de fibroblastos ativados e a deposição de colágeno por estes) é parte essencial do processo cicatricial, visto que esse processo é responsável por substituir a matriz extracelular por um tecido conjuntivo mais forte e elástico. Para a fibroplasia ser eficiente, é necessário que paralelamente a sua ocorrência se formem novos vasos sanguíneos (Balbino, Pereira \& Curi, 2005).

Por sua vez, acredita-se que o barbatimão influencie positivamente no processo cicatricial devido aos altos níveis de taninos e fenóis que este possui (Macedo, et al., 2007, Fonseca \& Librandi, 2008). Os taninos condensados promovem a cicatrização através da ligação com as proteínas do local da lesão, formando uma camada que protege o local da ferida, diminuindo sua permeabilidade e controlando o exsudato (Minatel, et al., 2010). A contribuição de fenóis para cicatrização está relacionada ao aumento da síntese de hidroxiprolina e hidroxilisina (principais aminoácidos envolvidos na cicatrização e 
precursores do colágeno), além de inibir a peroxidação lipídica, e aumentar a catalase, glutationa e superóxido dismutase (Zdunska, et al., 2018).

Albuquerque, et al., (2006) descrevem em seu estudo etnobotânico que dentre a vasta quantidade de plantas com propriedades terapêuticas comercializadas por vendedores de ervas medicinais dos mercados locais da cidade de Recife (Pernambuco-Brasil) encontra-se o barbatimão. Os comerciantes locais relatam que o barbatimão, além de ser usado para promover a cicatrização de feridas, também apresenta atividade anti-inflamatória, função adjuvante da cicatrização.

No Vale do Juruena, região do Mato Grosso (Brasil), a população utiliza de forma difundida o barbatimão em suas práticas de saúde. Dentre as finalidades medicinais descritas por Bieski, et al., (2015), encontra-se o uso para promover a cicatrização de feridas. Além do efeito cicatrizante, há outras propriedades do barbatimão conhecidas pela população do Vale do Juruena, que podem influenciar indiretamente no processo cicatricial, como o controle do Diabetes Mellitus, redução do processo inflamatório e ação antimicrobiana.

Na população ribeirinha da microrregião do Norte Araguaia (Mato Grosso-Brasil), os achados de Ribeiro, et al., (2017) se assemelham com os anteriormente descritos: nesta região o barbatimão é descrito como agente cicatrizante, sendo amplamente utilizando para promover a cicatrização de lesões cutâneas, inclusive as que apresentam sinais de infecção. Além desta propriedade, os ribeirinhos desta microrregião reconhecem que o barbatimão apresenta efeitos antimicrobianos e antiinflamatórios, efeitos estes que podem atuar de forma coadjuvante na propriedade cicatricial.

Em estudo realizado em uma microrregião no sul do Estado de Minas Gerais, levantaram-se as espécies mais utilizadas na medicina popular e como estas são manipuladas, sendo por sua vez, o barbatimão, uma das plantas mais utilizadas entre a população dessa localidade e com seu uso direcionado para diversas finalidades terapêuticas, como: hemorragias, diarreia, calvície e cicatrização (Rodrigues \& Carvalho, 2001).

Outra pesquisa, de finalidade etnobotânica e que corrobora com as discussões elencadas, é de Melo, (2011), que aborda essa planta como um vegetal com grande impacto no que diz respeito a sua utilização pelas comunidades, principalmente aquelas mais remotas, destacando a utilização do barbatimão para corrimentos vaginais, feridas abertas, úlceras estomacais e duodenais, leucorreia, afecções decorrentes de escorbuto e hemorragias. E coloca ainda que, a associação das cascas da planta com álcool, pode tornar o produto mais adstringente, resultando em efeitos mais significativos para o paciente.

É válido destacar e levando-se em consideração as informações colocadas anteriormente, é cada vez maior o interesse sobre plantas medicinais e suas possíveis aplicações terapêuticas. A medicina reconhecida como popular e o conhecimento específico sobre o uso de plantas é o resultado de séries de influências culturais, como a dos colonizadores europeus, dos indígenas e dos africanos. E esse fator histórico atrelado as raízes da cultura brasileira só fomentou a formação de um conjunto de conhecimentos acerca do uso de plantas, que atualmente congrega para a existência da fitoterapia popular, uma prática alternativa optada por milhares de brasileiros que não tem o devido acesso as práticas médicas oficiais em virtude dos altos custos, relacionados a procedimentos e medicamentos (Albuquerque \& Andrade, 2019).

Dessa forma, os saberes populares são de grande valia para nortear as descobertas ao se tratar do uso de tais plantas para fins medicinais, porém existem poucos produtos a base de barbatimão destinado à cicatrização de feridas cutâneas registrados em órgãos oficiais.

Este fato demonstra que o uso do barbatimão na cicatrização, sem a realização de testes de controle de qualidade, pode trazer riscos para a população, pois as preparações fitoterápicas, principalmente as de uso popular, podem possuir agentes contaminantes, substâncias adulterantes ou ambos e resultar na ineficiência do produto no processo cicatricial da lesão. Todos os trabalhos apresentados corroboram que o Barbatimão é utilizado no processo de cicatrização, em suas diversas fases, porém não sendo possível identificar para qual tipo, estágio ou etiologia de ferida o seu uso é mais adequado. 


\section{Considerações Finais}

Apesar das propriedades do barbatimão descritas na literatura, sua utilização ainda é pouco difundida no meio científico no processo de cicatrização de ferimentos e lesões, mesmo com o conhecimento popular acerca de sua aplicabilidade, os estudos pouco tratam a preparação, concentração, tempo de uso, interações com outras medicações e efeitos adversos acerca do barbatimão, dificultando sua avaliação quanto sua eficácia terapêutica.

Por fim, ressalta-se a importância da realização de novos estudos sobre as propriedades do barbatimão na cicatrização de feridas e lesões, haja vista que esse assunto poderá contribuir na disseminação do conhecimento científico sobre do uso de fitoterápicos.

\section{Referências}

Albuquerque, U. P. e Andrade, L. H. C (2019). Fitoterapia: uma Alternativa para Quem?. ttps://www.ufpe.br/documents/1192056/0/Fitoterapia +uma+alternativa+para+quem.docx.pdf/fe1c7916-113f-49d2-971f-400a8743653c.

Albuquerque, U. P.; Monteiro J. M.; Ramos, M. A. \& Amorim, ELC (2006). Medicinal and magic plants from a public market in northeastern. Brazil Journal of Ethnopharmacology, 110 (01), 76-91. Doi: https://doi.org/10.1016/j.jep.2006.09.010

Balbino, C. A.; Pereira, L. M. \& Curi, R. (2005). Mecanismos envolvidos na cicatrização: uma revisão. Brazilian Journal of Pharmaceutical Sciences, 41 (1). https://www.scielo.br/j/rbcf/a/HXZMLDrTL5B7mrRRqSsbtmh/?format=pdf

Bieski, I. G. C.; Leonti, M.; Arnason J. T.; ... \& Martins D. T. O. (2015). Ethnobotanical study of medicinal plants bypopulation of Valley of Juruena Region, LegalAmazon, Mato Grosso, Brazil. Journal of Ethnopharmacology, 173, 383-423. doi: https://doi.org/10.1016/j.jep.2015.07.025

Brasil. (2014). Ministério da Saúde. ANVISA. Monografia da espécie Stryphnodendronadstringens(mart.) Coville(Barbatimão). Brasilia. https://portalarquivos2.saude.gov.br/images/pdf/2014/novembro/25/Vers--o-cp-Stryphnodendron.pdf

Eurides, D.; Mazzanti, A.; Belleti, M. E.; Silva, L. A. F.; Fioravanti, M. C. S.; Neto, N. S. T.; Campos, V. A.; Lemos, R. C. \& Junior, P. L. S. (1996). Morfologia e Morfometria da Reparação Tecidual de Feridas Cutâneas de Camundongos Tratadas com Solução Aquosa de Barbatimão, v. 2 (1), 30-40. https://repositorio.bc.ufg.br/bitstream/ri/13477/5/Artigo\%20-\%20Duvaldo\%20Eurides\%20-\%20\%201996\%20.pdf

Farmacopeia Brasileira. (2011) Formulário de fitoterápicos, ANVISA, (1) 119.

Fonseca, P. \& Librandi, A. P. L. (2008). Avaliação das características físico-químicas e fitoquímicas de diferentes tinturas de barbatimão (Stryphnodedron barbartiman). Brazilian Journal of Pharmaceutical Sciences, 44, (2). doi: https://doi.org/10.1590/S1516-93322008000200012

Lima, T. C. D.; Cardoso, M. V.; Modesto, T.; Oliveira, A. L. B.; Silva, M. N. \& Monteiro, M. C. (2016). Breve Revisão Etnobotânica, Fitoquímica e Farmacologia de Stryphnodendronadstringens Utilizada na Amazônia. Revista Fitos, 10(3), 220-372. doi: 10.5935/2446-4775.20160025

Macedo, F. M. (2007). Determinação de CompostosFenólicos Totais em Barbatimão[Stryphnodendron adstringens (Mart) Coville]. Revista Brasileira de Biociências, 5 (2). https://seer.ufrgs.br/rbrasbioci/article/view/115382/62679

Medeiros, A. C. \& Dantas-Filho, A. M. (2017). Cicatrização das feridas cirúrgicas. Revista de Pesquisa Cirúrgica e Clínica, 7(2), 87-102.

Melo, J. A. (2011). Valorização da flora do cerrado com importância medicinal. Universidade de Brasília. Trabalho de Conclusão de Curso, Luziânia. 11-19. https://www.bdm.unb.br/bitstream/10483/1857/1/2011_JeaneAparecidaMelo.pdf

Mendes, k. D.s.; silveira, r. C. C. P. \& galvão, c. M. (2008). Revisão integrativa: método de pesquisa para a incorporação de evidências na saúde e na enfermagem. Texto contexto enferm, 17(4), 758-64.doi: Https://Doi.Org/10.1590/S0104-07072008000400018

Minatel, D. G.; Pereira, M. A. S.; Chiaratti, T. M.; Pasqualin, L.; Oliveira, J. C. N. \& Couto, L. B. (2010) Estudo clínico para validação da eficácia de pomada contendo barbatimão (Stryphnodendron adstringens (Mart.) Coville) na cicatrização de ulceras de decúbito. Rev Bras Med, 67(7), 250-56. http://bases.bireme.br/cgi-

bin/wxislind.exe/iah/online/?IsisScript=iah/iah.xis\&base=LILACS\&lang=p\&nextAction=lnk\&exprSearch=553884\&indexSearch=ID

Passaretti, T.; Guarnieri, A. P.; Filipini, R.; Alves, B. C. A. \& Fonseca, F. L. A. (2016). Eficácia do Uso do Barbatimão (Stryphnodendronbarbatiman) no Processo de Cicatrização em Lesões: uma Revisão de Literatura. ABCS Health Sci. 41(1), 51-54. Doi: http://dx.doi.org/10.7322/abcshs.v41i1.846

Pereira, C. Moreno, C. S. \& Carvalho, C. (2013). Usos Farmacológicos do Stryphnodendronadstringens(mar.) - barbatimão.Revista Panorâmica On-Line. Barra do Garças - MT, 15, 127 - 137. http://www.unieuro.edu.br/sitenovo/revistas/downloads/farmacia/cenarium_03_01.pdf

Ramalho, M. P.; Santos, S. L. F.; Castro, N. M.; Vasconcelos, L. M. O.; Morais, I. C. O. \& Pessoa, C. V. (2018). Plantas Medicinais no Processo de Cicatrização de Feridas: Revisão de Literatura.Rev. Expr. Catól. Saúde; 3 (2). doi: http://dx.doi.org/10.1590/1983-084X/12_178

Ribeiro, R. V. (2017). Ethnobotanical study of medicinal plants used by Ribeirinhos in the NorthAraguaia microregion, Mato Grosso, Brazil. Journal of Ethnopharmacology. doi: https://doi.org/10.1016/j.jep.2017.04.023

Rodrigues, V. E. G. \& Carvalho D. A. (2001). Levantamento etnobotânico de plantas medicinais no domínio do cerrado na região do Alto do Rio GrandeMinas Gerais. Ciênc. agrotec., Lavras, 25 (1), 102-123. https://www.agencia.cnptia.embrapa.br/recursos/FLO_Etnob_Cerrado_MGID-0zWHltLEGY.pdf 
Research, Society and Development, v. 10, n. 15, e394101522688, 2021

(CC BY 4.0) | ISSN 2525-3409 | DOI: http://dx.doi.org/10.33448/rsd-v10i15.22688

Souza, D. R. \& Rodrigues, E. C. A. M. S. (2016). Plantas Medicinais: Indicação de Raizeiros para o Tratamento de Feridas. RevBrasPromoç Saúde, Fortaleza, 29(2), 197-203. https://docs.bvsalud.org/biblioref/2017/02/827499/6-artigo-plantas-medicinais-diogo.pdf

Souza, M. T.; Silva, M. D. \& Carvalho, R. (2010). Revisão integrativa: o que é e como fazer. Einstein. 8(1), 102- 6. doi: https://doi.org/10.1590/S167945082010RW1134

Veiga Junior, V. F. (2008). Estudo do consumo de plantas medicinais na Região Centro-Norte do Estado do Rio de Janeiro: aceitação pelos profissionais de saúde e modo de uso pela população. Revista Brasileira de Farmacognosia, v. 18, n. 2, p. 308-313, 2008

Wang, r.; lechtenberg, m.; sendker, j.; petereit, f.; deters, a. \& hensel, a. (2013). Wound-healing plants from tcm: in vitro investigations on selected tcm plants and their influence on human dermal fibroblasts and keratinocytes. Fitoterapia, 84, 308-17. doi: https://doi.Org/10.1590/S0102-695x2008000200027

Zduńska, K. (2018). Antioxidant Properties of Ferulic Acid and Its Possible Application. Skin Pharmacol Physiol, 6 (31). doi: https://doi.org/10.1159/000491755 